\title{
Influência da adição de resíduo de cinzas de bagaço de cana-de-açúcar nas propriedades tecnológicas de cerâmica vermelha
}

\author{
Influence of the addition of sugarcane bagasse ash waste in \\ the technological properties of red ceramic
}

De Faria, K.C.P., Gurgel, R.F., De Holanda, J.N.F.

\author{
Universidade Estadual do Norte Fluminense, Grupo de Materiais Cerâmicos, Av. Alberto Lamego 2000, \\ 28013-602 Campos dos Goytacazes-RJ \\ e-mail: katiacpfaria@hotmail.com; rfguenf2009@hotmail.com; holanda@uenf.br
}

\begin{abstract}
RESUMO
O Brasil é o maior produtor mundial de açúcar e álcool proveniente de cana-de-açúcar. A indústria da canade-açúcar tem contribuído para o fortalecimento do agronegócio, desenvolvimento econômico e social do país. No entanto, o processo produtivo da indústria de cana-de-açúcar gera enormes quantidades de resíduos sólidos na forma de cinzas. Estes resíduos sólidos são de difícil disposição final e podem gerar problemas de poluição ambiental. Por esse motivo, devem-se buscar novas alternativas tecnológicas viáveis para o reuso ou reciclagem destes abundantes resíduos. Este trabalho tem como objetivo utilizar o resíduo de cinzas de bagaço de cana-de-açúcar como uma matéria-prima alternativa de baixo custo em mistura com argila caulinítica para obtenção de cerâmica vermelha. Foram estudadas cinco formulações com 0, 5, 10, 15 e 20 \% em peso de resíduo de bagaço de cana-de-açúcar misturado com argila caulinítica. As peças cerâmicas foram preparadas por prensagem uniaxial e sinterizadas a $1000{ }^{\circ} \mathrm{C}(24 \mathrm{~h}$ frio a frio). As peças sinterizadas foram caracterizadas em termos de retração linear, absorção de água, massa específica aparente, porosidade aparente e resistência à compressão. Ficou comprovado, após resultados dos ensaios realizados, que a incorporação de resíduos de cinzas de bagaço de cana-de-açúcar afeta significativamente as propriedades tecnológicas de cerâmica vermelha. Portanto, o resíduo de cinzas de bagaço de cana-de-açúcar pode ser usado como uma matéria-prima alternativa de baixo custo em cerâmica vermelha, desde que seja adicionado em quantidades moderadas.
\end{abstract}

Palavras-chave: Cinzas, Bagaço de Cana-de-Açúcar, Cerâmica Vermelha, Reciclagem.

\begin{abstract}
Brazil is the world's largest producer of sugar and ethanol from sugarcane. The sugarcane industry has contributed to the strengthening of agribusiness, as well as economic and social development of the country. However, the production process of the sugarcane industry generates huge amounts of solid wastes in the form of ashes. These solid wastes have difficult disposal and can lead to problems of environmental pollution. Therefore, one should look for new alternative technologies viable for reuse or recycling of these abundant wastes. The essay's goal is to evaluate the use of sugarcane bagasse ash waste as a low cost alternative raw material mixed with clay for production of red ceramic. Five formulations were studied with 0, 5, 10, 15 and 20 wt.\% of waste mixed with kaolinitic clay. Ceramic pieces were prepared by uniaxial pressing and sintered at $1000{ }^{\circ} \mathrm{C}$ (24 cold cold). The pieces were characterized in terms of linear shrinkage, water absorption, apparent density, apparent porosity and compressive strength. It was proven, after results of tests performed, the incorporation of sugarcane bagasse ash waste significantly affects the technological properties of red ceramic. Therefore, the sugarcane bagasse ash waste can be used as an alternative raw material in red ceramic, since it is added in moderate amounts.
\end{abstract}

Keywords: Ashes, Sugarcane Bagasse, Red Ceramic, Recycling. 


\section{INTRODUÇÃO}

No Brasil a indústria da cana-de-açúcar é um os setores da agroindústria que mais se destacam em área de plantio e em produção. Estima-se que cerca de 15 \% dos solos agricultáveis do Brasil seja destinado ao plantio de cana-de-açúcar para produção de açúcar e álcool. O alto crescimento da indústria da cana-deaçúcar se deve em parte ao forte programa nacional de produção de álcool combustível denominado de Proálcool. A indústria da cana-de-açúcar, sem dúvida, tem contribuído para o fortalecimento do agronegócio, desenvolvimento econômico e social do país. No entanto, o processo produtivo da indústria de cana-deaçúcar gera enormes quantidades de resíduos sólidos na forma de cinzas [1].

Os resíduos sólidos de cinzas de bagaço de cana-de-açúcar são gerados a partir da queima do bagaço de cana-de-açúcar nas caldeiras geradoras de vapor das usinas. O bagaço de cana-de-açúcar é usado para cogeração de energia em substituição ao óleo combustível e outros energéticos. A quantidade de resíduo de cinzas de bagaço de cana-de-açúcar (RCBCA) gerada corresponde à cerca de $0,5 \%$ em peso, em relação à massa inicial do bagaço. Estima-se que no Brasil sejam produzidas nas usinas de açúcar e álcool cerca de 1.200.000 toneladas/ano de RCBCA [2-4]. Por outro lado, o RCBCA não tem uma utilização definida para a indústria da cana-de-açúcar. Por este motivo, o RCBCA vem sendo utilizado, por exemplo, como corretivo de solo sem obedecer qualquer parâmetro técnico [5].

O RCBCA apresenta em sua composição alto teor de sílica $\left(\mathrm{SiO}_{2}\right)$, além de pequenas quantidades de óxidos de $\mathrm{Al}, \mathrm{Fe}, \mathrm{Ca}, \mathrm{Mg}$ e $\mathrm{K}[1,6]$. Isto significa que do ponto de vista químico o RCBCA contém quantidades apreciáveis de óxidos presentes nas argilas usadas na fabricação de cerâmica vermelha. Além disso, por apresentar alto teor de sílica o RCBCA é considerado um material não plástico. De forma que estas características tornam o RCBCA atrativo como uma matéria-prima alternativa para fabricação de materiais de cerâmica vermelha. De fato as massas argilosas usadas em cerâmica vermelha são misturas de materiais plásticos e não plásticos que apresentam larga variabilidade em termos de composição química e mineralógica [7, 8]. Por outro lado, apesar de sua importância constata-se que pouca atenção tem sido dada no Brasil à incorporação deste abundante resíduo em cerâmica vermelha.

Neste contexto, o objetivo principal deste trabalho é avaliar as propriedades tecnológicas de cerâmica vermelha incorporada com resíduo de cinzas de bagaço de cana-de-açúcar. Este trabalho busca não somente contribuir com uma solução tecnológica viável para o destino final deste abundante resíduo, mas também estudar a influência da adição do RCBCA nas propriedades da cerâmica vermelha e determinar qual a quantidade adequada de resíduo a ser incorporada na formulação argilosa.

\section{MATERIAIS E MÉTODOS}

As matérias-primas usadas neste trabalho foram uma massa argilosa vermelha usada na fabricação de blocos cerâmicos (tijolos furados), e um resíduo de cinzas de bagaço de cana-de-açúcar (RCBCA) provenientes da região de Campos dos Goytacazes-RJ. Neste trabalho foi preparada uma série de misturas massa argilosa/RCBCA contendo até $20 \%$ em peso de RCBCA (Tabela 1). A massa argilosa isenta de resíduo (amostra MK0 - 100 \% de argila vermelha) é considerada a amostra de referência.

Tabela 1: Composição das massas cerâmicas estudadas (\% em peso).

\begin{tabular}{c|c|c}
\hline \multirow{2}{*}{ FORMULAÇÃO } & \multicolumn{2}{|c}{ MATÉRIAS-PRIMAS } \\
\cline { 2 - 3 } & MASSA ARGILOSA & RCBCA \\
\hline MK0 & 100 & 0 \\
\hline MK5 & 95 & 5 \\
\hline MK10 & 90 & 10 \\
\hline MK15 & 85 & 15 \\
\hline MK20 & 80 & 20 \\
\hline
\end{tabular}

A amostra de RCBCA in natura em forma de pó foi submetida a processo de beneficiamento com o objetivo de eliminar as impurezas grosseiras indesejáveis, como resto de folhas e bagaço de cana-de-açúcar não queimada. A massa argilosa vermelha passou pelo processo de secagem em estufa a $110{ }^{\circ} \mathrm{C}$. Em seguida as matérias-primas foram passadas em peneira com abertura de 42 mesh (ASTM $355 \mu \mathrm{m}$ ). A composição 
química das matérias-primas usadas neste trabalho é apresentada na Tabela 2. A amostra de RCBCA apresenta como principais fases cristalinas: quartzo e Cristobalita [6].

Tabela 2: Composição química das matérias-primas usadas (\% em peso).

\begin{tabular}{c|c|c}
\hline ÓXIDOS & ARGILA & RCBCA \\
\hline $\mathrm{SiO}_{2}$ & 46,95 & 61,59 \\
\hline $\mathrm{Al}_{2} \mathrm{O}_{3}$ & 36,19 & 5,92 \\
\hline $\mathrm{Fe}_{2} \mathrm{O}_{3}$ & 9,73 & 7,36 \\
\hline $\mathrm{SO}_{3}$ & 1,86 & 0,42 \\
\hline $\mathrm{TiO}_{2}$ & 1,66 & 2,46 \\
\hline $\mathrm{CaO}$ & 0,48 & 5,00 \\
\hline $\mathrm{MnO}$ & 0,11 & 0,10 \\
\hline $\mathrm{K}_{2} \mathrm{O}$ & 2,82 & 6,22 \\
\hline $\mathrm{ZrO}$ & 0,03 & 0,17 \\
\hline $\mathrm{V}_{2} \mathrm{O}_{5}$ & 0,12 & 0,10 \\
\hline $\mathrm{ZnO}$ & - & 0,10 \\
\hline $\mathrm{NiO}$ & - & 0,03 \\
\hline $\mathrm{SrO}$ & - & 0,02 \\
\hline $\mathrm{P.F}$. & 10,40 & 9,7 \\
\hline
\end{tabular}

As matérias-primas secas nas proporções de acordo com a Tabela 1 foram misturadas num misturador cilíndrico de laboratório (Gardelin, modelo 1A) durante $30 \mathrm{~min}$, e depois classificadas por peneiramento para $<42$ mesh. Em seguida as massas cerâmicas foram umidificadas para cerca de 7 \% em peso de água, e mantidas em sacos plásticos fechados em dessecador por $24 \mathrm{~h}$.

As massas cerâmicas argilosas foram submetidas à prensagem uniaxial com ação única do pistão a 21 MPa, utilizando-se uma matriz de aço de cavidade cilíndrica $(\phi=2,5 \mathrm{~cm})$. As peças cerâmicas compactadas foram submetidas a processo de secagem em estufa a $110{ }^{\circ} \mathrm{C}$ por um período de $24 \mathrm{~h}$. $\mathrm{O}$ processo de queima das peças de cerâmica vermelha secas foi realizado num forno elétrico tipo mufla numa temperatura de 1000 ${ }^{\circ} \mathrm{C}$. Foi empregado um ciclo de queima lento ( $24 \mathrm{~h}$ frio a frio). O tempo de permanência das peças na temperatura de patamar foi de $2 \mathrm{~h}$.

As peças de cerâmica vermelha queimadas foram caracterizadas em termos de retração linear, absorção de água, massa específica aparente, porosidade aparente e resistência à compressão. A retração linear foi determinada utilizando um paquímetro digital com precisão de $\pm 0,01 \mathrm{~mm}$, para medição dos comprimentos dos diâmetros antes e após queima. A absorção de água, massa específica aparente e a porosidade aparente das peças cerâmicas foram obtidas de acordo com procedimentos padronizados ASTM 373 [9]. A resistência à compressão diametral foi determinada com o auxílio de uma máquina de ensaios universal (EMIC, modelo DL100/100kN) com velocidades de carregamento de $0,5 \mathrm{~mm} / \mathrm{min}$ de acordo com a expressão [10,11]:

$$
R c_{Q}=\frac{2 F}{\pi d t}
$$

Onde:

$\mathrm{Rc}_{\mathrm{q}}$ - resistência a compressão diametral, em MPa;

$\mathrm{F}$ - carga de ruptura, em $\mathrm{N}$;

d - diâmetro das peças queimadas, em mm;

$\mathrm{t}$ - altura das peças cilíndricas, em mm. 


\section{RESULTADOS E DISCUSSÃO}

A Figura 1 apresenta o comportamento da retração linear de queima das peças de cerâmica vermelha em função do teor de RCBCA incorporado. Nota-se uma leve diminuição na retração linear para adições de RCBCA a partir de $10 \%$ em peso (composições MK10, MK15 e MK20). Isto está fundamentalmente relacionado ao fato do RCBCA apresentar alta concentração de quartzo, conforme mostrado na Tabela 2. De fato o RCBCA é um material não plástico [1, 6], o qual é responsável pela redução da plasticidade global do mix massa argilosa/RCBCA. De forma que a adição do RCBCA tem o efeito benéfico de melhorar a estabilidade dimensional das peças de cerâmica vermelha. Pode-se observar também que a retração linear de queima está na faixa de 2,00 - 2,79 \%. Estes valores estão dentro da faixa ótima para produção industrial de cerâmica vermelha.

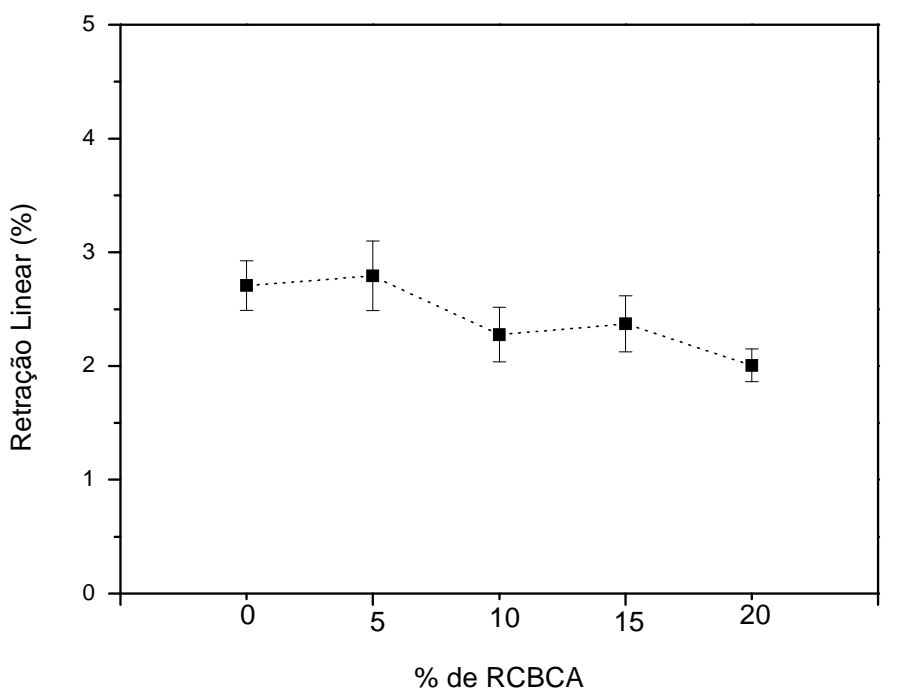

Figura 1: Retração linear das peças cerâmicas queimadas a $1000^{\circ} \mathrm{C}$.

A Figura 2 apresenta o comportamento da absorção de água das peças de cerâmica vermelha. A absorção de água é uma propriedade física que está associada à porosidade aberta e microestrutura do material sinterizado. Nota-se que incorporação de até $10 \%$ de RCBCA (composições MK5 e MK10) praticamente não acarretou alteração nos valores de absorção de água. Os resultados mostram que adições acima de 10 \% de RCBCA tende a aumentar a absorção de água das peças cerâmicas. A explicação para isto pode estar relacionada ao fato do RCBCA conter em sua composição quantidade apreciável de matéria orgânica. Durante o processo de queima ocorre à decomposição da matéria orgânica, o qual contribui para gerar poros abertos na estrutura do material. Isto significa que altas concentrações de RCBCA provocarão um aumento da absorção de água (porosidade aberta) das peças de cerâmica vermelha. Isto deve ser evitado uma vez que a absorção de água é uma propriedade técnica usada para especificar a qualidade do produto de cerâmica vermelha. Por exemplo, os tijolos maciços e blocos cerâmicos (tijolos furados) devem apresentar valor de absorção de água $<25$ \%. A porosidade aparente das peças de cerâmica vermelha contendo RCBCA, como mostrado na Figura 3, seguiu o mesmo comportamento da absorção de água. 


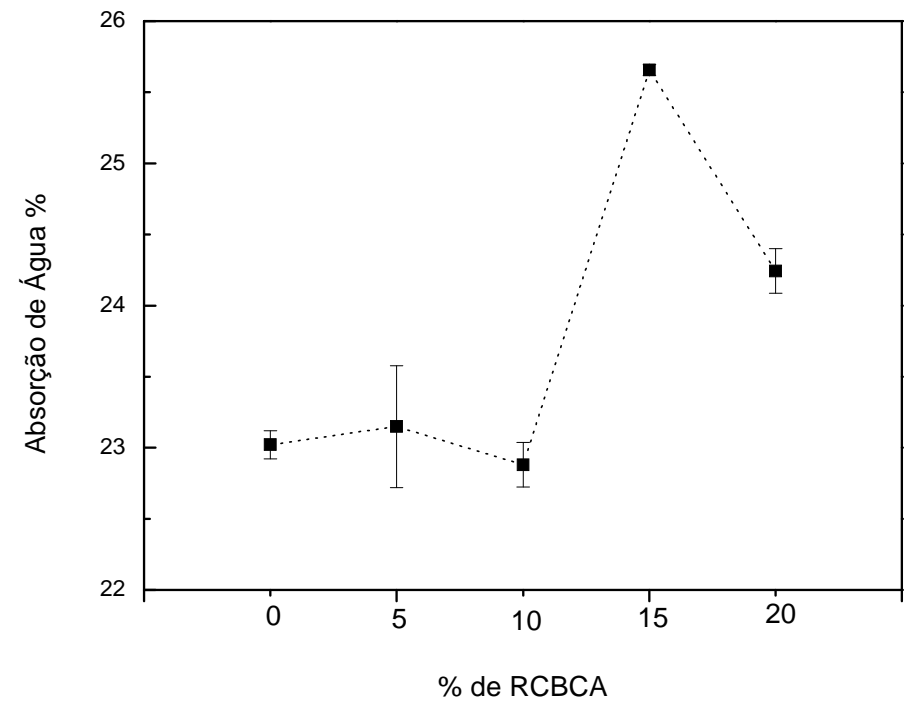

Figura 2: Absorção de água das peças cerâmicas queimadas a $1000^{\circ} \mathrm{C}$.

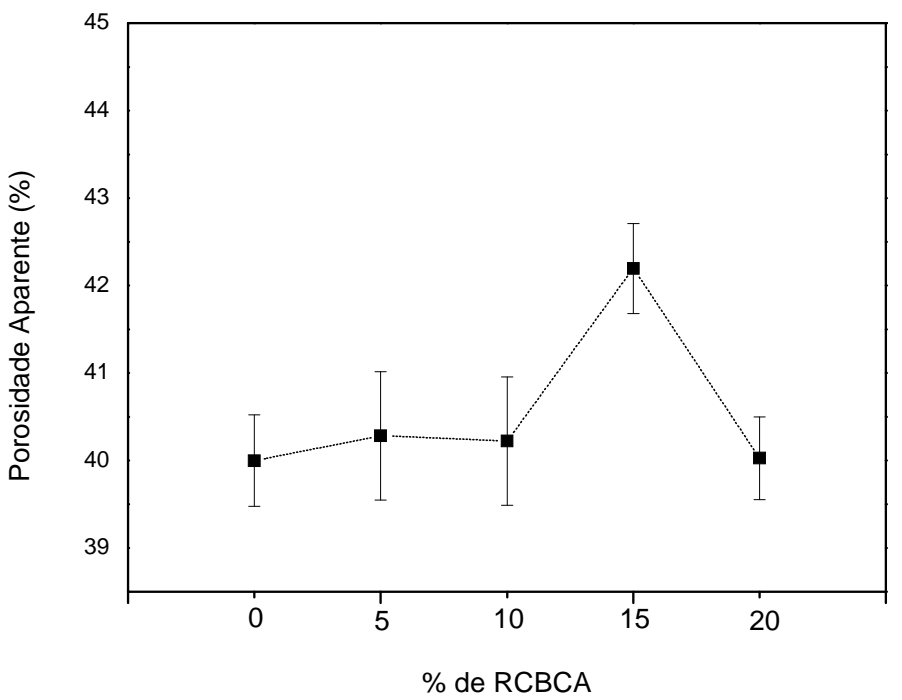

Figura 3: Porosidade aparente das peças cerâmicas queimadas a $1000^{\circ} \mathrm{C}$.

A Figura 4 apresenta a massa específica aparente das peças cerâmicas queimada a $1000{ }^{\circ} \mathrm{C}$ em função do teor de RCBCA adicionado. Observa-se que o efeito do RCBCA é o de diminuir a densificação das peças cerâmicas. Isto está de acordo com os resultados de absorção de água (Fig. 2) e porosidade aparente (Fig. 3). 


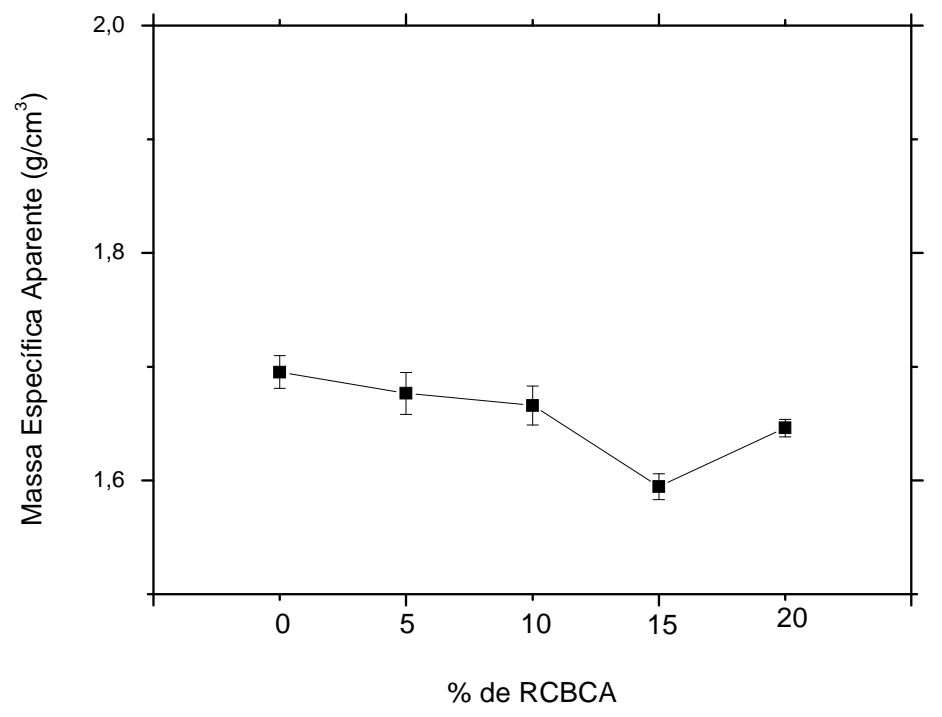

Figura 4: Massa específica aparente das peças cerâmicas queimadas a $1000^{\circ} \mathrm{C}$.

A resistência à compressão diametral das peças cerâmicas queimadas é mostrada na Figura 5. Pode-se observar que o efeito da incorporação do resíduo foi o de diminuir a resistência mecânica das peças de cerâmica vermelha.

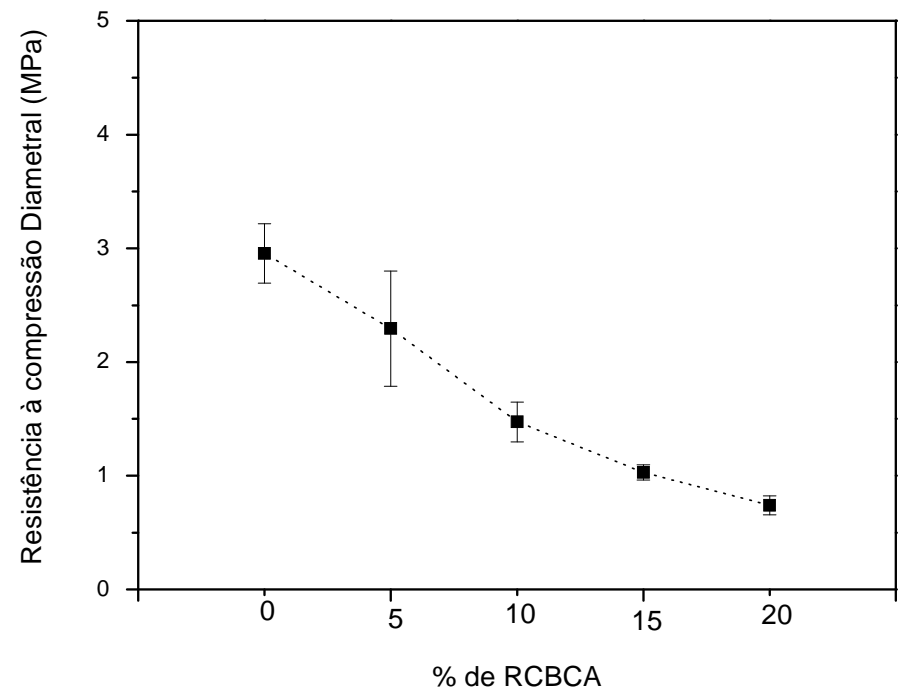

Figura 5: Resistência à compressão diametral das peças cerâmicas queimadas a $1000^{\circ} \mathrm{C}$.

O efeito deletério do RCBCA na resistência a compressão diametral está fundamentalmente relacionado ao seu principal componente mineralógico, no caso o quartzo. A rigor o quartzo atua como defeito crítico na estrutura sinterizada da peça cerâmica [3]. Este resultado demonstra categoricamente que se deve evitar o uso de altas concentrações de RCBCA nas formulações argilosas para cerâmica vermelha. 


\section{CONCLUSÕES}

Os resultados deste trabalho demonstram que o resíduo de cinzas de bagaço de cana-de-açúcar (RCBCA) tem grande potencial para ser utilizado como uma matéria-prima alternativa na fabricação de produtos de cerâmica vermelha. No entanto, a incorporação de RCBCA na formulação argilosa tende a influenciar fortemente as propriedades tecnológicas (retração linear, absorção de água, massa específica aparente, porosidade aparente e resistência à compressão) das peças cerâmicas. Foi observado que a incorporação de RCBCA resultou no efeito positivo de diminuir a retração linear e contribuiu para uma melhor estabilidade dimensional das peças cerâmicas. Por outro lado, a incorporação de RCBCA também aumentou a absorção de água (porosidade aberta) e diminuiu a resistência mecânica das peças cerâmicas sinterizadas. Estes efeitos estão fundamentalmente relacionados às composições química e mineralógica do RCBCA, que é rico em quartzo e matéria orgânica. Por este motivo, o uso de RCBCA em cerâmica vermelha deve ser feito em quantidades moderadas. Os resultados deste trabalho indicam o uso de até $10 \%$ em peso do RCBCA utilizado como substituto de argila natural na fabricação de cerâmica vermelha.

\section{AGRADECIMENTOS}

Os autores gostariam de agradecer ao CNPq e FAPERJ pelo apoio financeiro, a Usina Sapucaia pelo fornecimento do resíduo de bagaço de cana-de-açúcar e a Cerâmica São José pelo fornecimento da massa argilosa.

\section{BIBLIOGRAFIA}

[1] GURGEL, R.F., “Caracterização físico-química e térmica de resíduo de cinza de bagaço de cana-deaçúcar”. Monografia de Graduação, UENF-CEMM, Campos dos Goytacazes, 2009.

[2] FREITAS, E.S., Caracterização de cinza de bagaço da cana-de-açúcar do município de Campos dos Goytacazes para uso na construção civil, Dissertação de M.Sc., UENF-PPGEC, Campos dos Goytacazes, 2005.

[3] TEIXEIRA, R.S., SOUZA, A.E., SANTOS, G.T.A., et al., "Sugarcane bagasse ash as a potential quartz replacement in red ceramic”, Journal of the American Ceramic Society, v.91, n.6, pp.1883-1887, 2008.

[4] BORLINI, M.C., MENDONÇA, J.L.C.C., PINATTI, D.G.,et al., "Cerâmica com cinza de bagaço de cana-de-açúcar : avaliação da influência da cinza e da granulometria nas propriedades físicas e mecânicas”, Anais do $17^{\circ}$ CBECIMAT, Foz do Iguaçu, pp. 2033-2041, novembro, 2006.

[5] CINCOTTO, M.A., Utilização dos subprodutos e resíduos na Indústria da construção civil. Tecnologia das Edificações, PINI, São Paulo, 1988.

[6] FARIA, K.C.P.,GURGEL, R.F., HOLANDA, J.N.F., “Characterization of sugarcane bagasse ash for use in ceramic bodies”, Proceedings of $7^{\text {th }}$ PETCH 09, Atibaia, pp. 1292-1295, 2009.

[7] SANTOS, P.S., Ciência e tecnologia das argilas, 2a Edição, São Paulo, Edgard Blucher, v.1, pp.405, 1989.

[8] GOMES, C.F., “Argilas: o que são e para que servem”. In: Fundação Calouste Gulbenkian, pp.457, Lisboa,1988.

[9] ASTM C 373, Test method for water absorption, bulk density, apparent porosity, and apparent specific gravity of fired whiteware products, 1994.

[10] FETT, T., T-stress in rectangular plates and circular disks. Eng. Frac. Mechanics. v. 60, pp. 631-652, 1998.

[11] CHEN, F., SUN, Z., XU, J., "Mode I fracture analysis of the double edge cracked Brazilian disk using a weight function method”, International Journal of Rock Mechanics and Mining Sciences, v.38, pp. 475-479, 2001. 